\title{
UNSUPERVISED TIME REVERSAL BASED MICROWAVE IMAGING FOR BREAST CANCER DETECTION
}

\author{
Mohmmad H. S. Sajjadieh and Amir Asif \\ York University \\ Department of Computer Science \& Engineering \\ 4700 Keele Street, Toronto, Ontario, CANADA M3J 1P3 \\ $\{$ mohammad,asif $\} @$ cse.yorku.ca
}

\begin{abstract}
Microwave breast imaging is performed by illuminating the breast tissues with a short pulse of microwaves and processing the reflections (backscatter) to create a pseudospectrum that detects the presence of the breast tumours specifying their locations. An important step in such breast cancer detection techniques is the backscatter preconditioning step for effective suppression of the clutter - signals arising from scattering mechanisms other than the tumor including the antenna reverberations and reflections from the skin-breast interface and chest wall. The paper proposes a new clutter suppression algorithm that successfully isolates the tumour response from the overall (tumour and clutter) response. The proposed DAF/EDF approach is based on a combination of the data adaptive filter (DAF) and the envelope detection filter (EDF), and does not require any prior training. The DAF/EDF algorithm is then coupled with the time reversal (TR) array imaging approaches $[1,2,3]$ and tested by running finite difference, time difference (FDTD) electromagnetic simulations based on the magnetic resonance imaging (MRI) data of the human breast. Our results demonstrate the effectiveness of the $\mathrm{DAF} / \mathrm{EDF}$ algorithm for microwave breast cancer detection.
\end{abstract}

Index Terms - Time Reversal, Breast Cancer Detection, Microwave Imaging, Clutter Suppression, Array Processing.

\section{INTRODUCTION}

In backscatter imaging, several microwave emitters illuminate the breast and the resulting backscatter waveform is recorded by a specialized antenna array. Malignant breast tumours exhibit significantly different electrical properties from those of the healthy breast tissues $[4,5]$. Consequently, a cancerous tumour produces a stronger electromagnetic backscatter, which can then possibly be processed using standard array processing approaches to locate the tumour. An important initial step in the breast cancer detection algorithms is backscatter pre-conditioning for effective suppression of the clutter. In the context of breast cancer detection, clutter is defined as signals $\mathbf{k}_{c}[n]$ arising from scattering mechanisms other than the tumor, which include the antenna reverberations and reflections from the skin-breast interface and chest wall. Effective suppression of the clutter is necessary to accurately detect and localize the breast tumors. Background subtraction procedures [6] typically used in radar applications for estimating the target response $\mathbf{k}_{t}[n]$ from the overall (target and clutter response) $\mathbf{k}_{c+t}[n]$ assumes a linear system and requires knowledge of the clutter response, i.e.,

$$
\mathbf{k}_{t}[n]=\mathbf{k}_{c+t}[n]-\mathbf{k}_{c}[n] .
$$

It is, therefore, necessary to have prior knowledge of the response of the normal breast tissues without tumour in an environment identical to the one in which the examination for finding anomalies is later conducted. This is not possible even in screening situations because of the variations in the normal tissues and their growth over time. Therefore, the background subtraction procedure, if used, introduces unwanted distortions in the target response. To decompose the overall response $\mathbf{k}_{c+t}[n]$ into the target response $\mathbf{k}_{t}[n]$ and the clutter response $\mathbf{k}_{c}[n]$ without a training step, we present a filtering algorithm, namely the data adaptive filter/envelope detection filter (DAF/EDF). This is the first contribution of the paper. Estimation of the target response in a cluttered medium has also been considered in $[7,9]$. However, these approaches can not be applied to breast cancer detection. For example, the algorithm proposed in [9] uses the matched filter technique to extract the tumour response but it requires a good estimation of the clutter template as an input. The proposed DAF/EDF does not require the template for the clutter response and is, therefore, more suitable for breast cancer detection.

A second problem arises due to multipath, the phenomena in which the backscatter waveform reaches the sensor array via two or more paths. Unlike X-rays, which are non-diffractive and travel in straight lines, microwave propagation in breast tissues is characterized by refraction and multipath effects - typically considered detrimental in standard signal processing approaches. Time reversal (TR) offers an alternative paradigm and treats multipath positively by tailoring the received backscatters to the scattering properties of the medium. To overcome the multipath problem, we couple the DAF/EDF clutter suppression algorithm with the TR imaging approaches $[1,2,3]$. When tested using the finite difference, time difference (FDTD) electromagnetic simulation environment, derived from the magnetic resonance imaging (MRI) data of the human breast, our approach shows great promise for breast cancer detection.

The paper is organized as follows. Section 2 defines the notation and reviews the data adaptive filter [7]. The proposed DAF/EDF algorithm is presented in Section 3, while Section 4 presents the detection results based on the electromagnetic breast model [1] derived from an MRI. Finally, Section 5 concludes the paper.

\section{THE DATA ADAPTIVE FILTER ALGORITHM}

Fig. 1 shows the setup, comprising of two antenna arrays (Arrays $A$ and $B$ ) with equal number $N$ of elements, used for breast cancer detection. The elements of Array $A$ are referred to as $\left(1 \leq A_{i} \leq N\right)$ and similarly, $\left(1 \leq B_{i} \leq N\right)$ for Array $B$. As each element of Array $A$ probes the breast tissue, all elements in Array $B$ record the mixed backscatter waveforms $\mathbf{k}_{c+t}^{\left(B_{i}\right)}[n]$ resulting in a $(N \times N)$ matrix, re- 


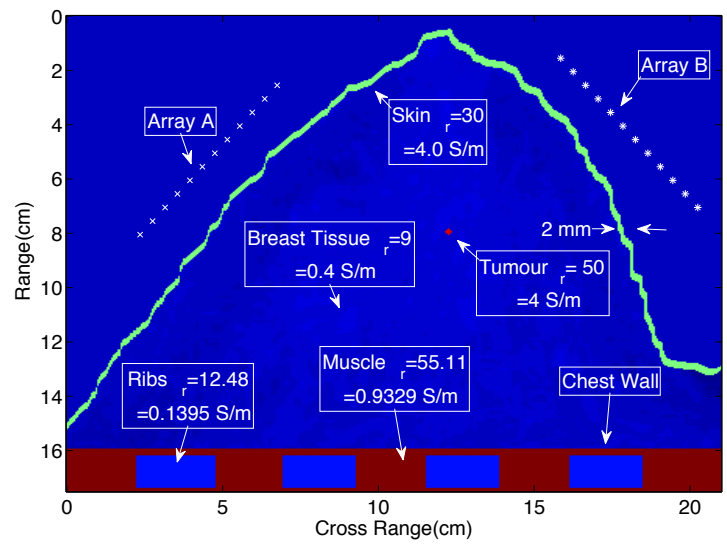

Fig. 1: The EM breast model derived from the MRI of a patient with the proposed antenna geometry (Arrays $A$ and $B$ ) parallel to the chest walls. Normally, the antenna arrays are placed well above the breast with the arrays parallel to the chest wall.

ferred to as the multistatic matrix. Assume that we want to remove the effect of clutter from the first mixed recorded response $\mathbf{k}_{c+t}^{(1)}[n]$ recorded at element $B_{1}$ of the receiving array, when the first element $A_{1}$ in the transmitter array probes the reference medium. To estimate the clutter response for the first element, the remaining overall responses $\left[\mathbf{k}_{c+t}^{(2)}, \cdots, \mathbf{k}_{c+t}^{(N)}\right]$ recorded when element $A_{1}$ probes the medium are used. The data adaptive filter (DAF) [7] assumes a delay of $J$ samples between the probing signal and its clutter response recorded at the neighboring elements of the receiving array. The actual value of the delay $J$ is determined experimentally. To estimate the clutter contribution $k_{c}^{(1)}[n]$ at sample value $n$ of $\mathbf{k}_{c+t}^{(1)}[n]$, the following $(N-1)$ vectors of length $(2 J+1)$ are defined

$$
\begin{aligned}
& \forall \quad 2 \leq i \leq N: \\
& \mathbf{b}_{i}[n]=\left[k_{c+t}^{(i)}[n-J], \cdots, k_{c+t}^{(i)}[n], \cdots, k_{c+t}^{(i)}[n+J]\right]^{T} .
\end{aligned}
$$

By concatenating all vectors $\mathbf{b}_{i}[n],(2 \leq i \leq N)$, we get

$$
\mathbf{b}_{2 N}[n]=\left[\mathbf{b}_{2}^{T}[n], \cdots, \mathbf{b}_{N}^{T}[n]\right]^{T},
$$

of length $(N-1)(2 J+1)$. By selecting the value of $n$ appropriately, $\mathbf{b}_{2 N}$ can be constructed such that it includes the clutter response primarily. The filter coefficients of the DAF are then obtained by solving the following minimization problem

$$
\min _{\mathbf{q}_{2 N}} \sum_{n=n_{0}}^{n_{0}+m-1}|k_{c+t}^{(1)}[n]-\underbrace{\mathbf{q}_{2 N}^{T}[n] \mathbf{b}_{2 N}[n]}_{\hat{k}_{c}^{(1)}[n]}|^{2},
$$

where $\mathbf{q}_{2 N}^{T}[n]$, for $(0 \leq n \leq(N-1)(2 J+1)-1)$, is the coefficient filter vector of the DAF. Note that the applied window $\left(n_{0} \leq n \leq\right.$ $\left.\left(n_{0}+m-1\right)\right)$ used for the summation operation is picked from a region within the mixed response, which constitute primarily of the clutter response. The duration of the window corresponds to the length of clutter response which is determined experimentally through a training step. The LMS solution [7] to Eq. (4) is given by

$$
\mathbf{q}_{2 N}=\mathbf{R}^{-1} \mathbf{p}
$$

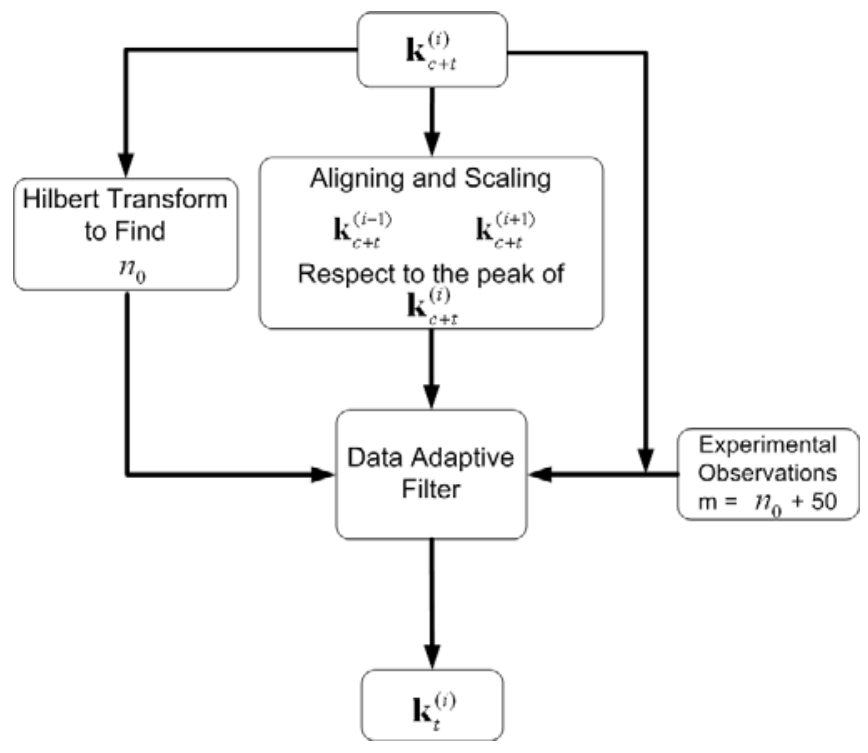

Fig. 2: The DAF/EDF algorithm.

$$
\begin{array}{ll}
\text { where } & \mathbf{R}=\frac{1}{m} \sum_{n=n_{0}}^{n_{0}+m-1} \mathbf{b}_{2 N}[n] \mathbf{b}_{2 N}{ }^{T}[n], \\
\text { and } & \mathbf{p}=\frac{1}{m} \sum_{n=n_{0}}^{n_{0}+m-1} \mathbf{b}_{2 N}[n] k_{c+t}^{(1)}[n] .
\end{array}
$$

Since the recorded backscatters $\mathbf{k}_{c+t}^{(1)}[n]$ with the selected range, $\left(n_{0} \leq n \leq n_{0}+m-1\right)$, are similar in magnitude at all receivers, matrix $\mathbf{R}$ is ill-conditioned and, therefore, singular. The inverse of $\mathbf{R}$ is computed from its $s$ significant eigenvalues $\lambda_{i=1}^{s}$ and the corresponding eigenvectors $\mathbf{u}_{i}^{s}$ as follows

$$
\widehat{\mathbf{R}}_{s}=\sum_{i=1}^{s} \frac{1}{\lambda_{i}} \mathbf{u}_{i} \mathbf{u}_{i}^{T} .
$$

The clutter artifacts due to skin, breast inhomogeneities, and chest wall can be removed from the $n$ 'th sample in $\mathbf{k}_{c+t}^{(1)}$ using

$$
\hat{k}_{t}^{(1)}[n]=k_{c+t}^{(1)}[n]-\mathbf{q}_{2 N}^{T}[n] \mathbf{b}_{2 N}[n] .
$$

The above algorithm is repeated for all mixed responses $\mathbf{k}_{c+t}^{(i)}[n]$ recorded at different elements of the receiving array when successive elements of the transmitting array probes the medium. Likewise, the algorithm is repeated for all other transmitter-receiver pairs to estimate the target response for all pairs.

There are certain drawbacks with the DAF approach. First, it requires a training step, where coefficients $n_{0}$ and $m$ are determined empirically. Without the training step, there is no way to define the temporal window $\left[n_{0}, \cdots, n_{0}+m-1\right]$ such that it corresponds only to the clutter. For patients belonging to different age groups and ethnicity, the values of these variables can be quite different. The geometry of Arrays $A$ and $B$ with respect to the breast also affects these values. Further, there is a hidden variable $s$ corresponding to the number of significant eigenvalues, which also varies from one patient to another and is, therefore, difficult to generalize solely from the singular value decomposition (SVD). Next, we propose a new filter based on the DAF, which determines parameters $n_{0}, m$, and $s$ without the need of a training step. 


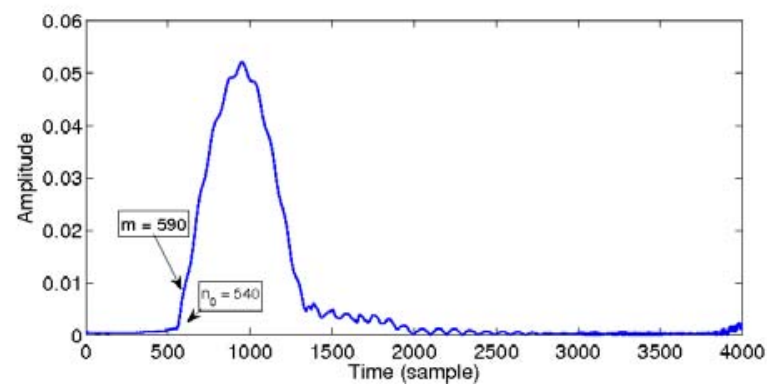

Fig. 3: The output of the low pass filter on the mixed response to determine $n_{0}$ and $m$ parameters.

\section{THE DAF/EDF ALGORITHM}

As illustrated in Fig. 2, the proposed DAF/EDF algorithm consists of the following four steps.

1. Starting Indices $n_{0}$ and $m$ : Our simulations show that the starting location of the tumour response $\mathbf{k}_{t}[n]$ in the overall response $\mathbf{k}_{c+t}[n]$ depends on the geometry of Arrays $A$ and $B$ with respect to the chest wall and the location of the tumour. For example, when both arrays are parallel to the chest wall and the tumour location is in the middle of the breast, the starting index of the tumour response $\mathbf{k}_{t}[n]$ is well after the peak of the clutter reflection. Although the separation of these two signals using this parallel array geometry provides a way to select the starting index $n_{0}$ of the temporal window, the relative magnitude of the tumour response compared to the clutter response is low such that it is not recognizable even if the starting index $n_{0}$ and length $m$ of the window are known. I propose another geometry for Array $A$ and $B$ which is closer to the breast and parallel to the breast skin (Fig. 1). Use of this geometry makes the magnitude of the tumour response comparable to the clutter response. However, this results in an overlap between the tumour and clutter responses. Our simulations show that there is a delay between the starting indices of the clutter response $\mathbf{k}_{c}[n]$ and tumour response $\mathbf{k}_{t}[n]$, which can be used to calculate the DAF filter coefficients. Based on our simulations, the delay is never less than 50 samples, which implies $m=n_{0}+50$. Referred in the literature as the time of arrival (TOA), the delay depends on the location of the tumour with respect to the skin and receiving elements. The TOA can, therefore, be determined by dividing the breast in different regions and noting the time difference between the relection from the skin and the reflection from the centroid of the region. The minimum of the values observed is then taken as the value for the delay. In order to find the starting index $n_{0}$, all mixed responses $\mathbf{k}_{c+t}[n]$ are passed through a low pass filter to derive the envelope of the signals. The first peak in the envelope corresponds to the sample where the energy of the signal starts to grow. Since we expect the primary portion of this rise to be related to the clutter because the skin is closer to receiver antennas, the starting index $n_{0}$ is given by sample corresponding to the rise. We use Hilbert's algorithm implemented by Kolmogorov [8] for envelope detection. Fig. 3 shows the output of the envelope detector for the overall response $\mathbf{k}_{c+t}[n]$ shown in Fig. 4(a).

2. Clutter Signature: For the $i$ 'th receiver, $(2 \leq i \leq N-1)$,

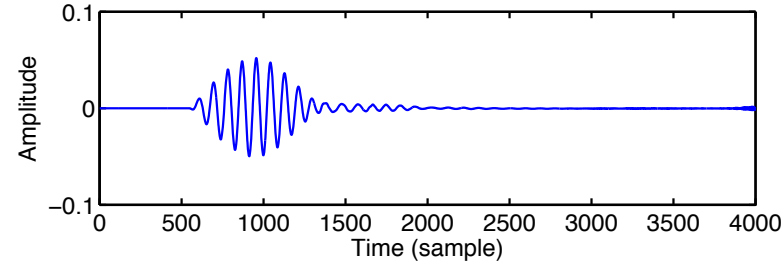

(a) $\mathbf{k}_{c+t}[n]$

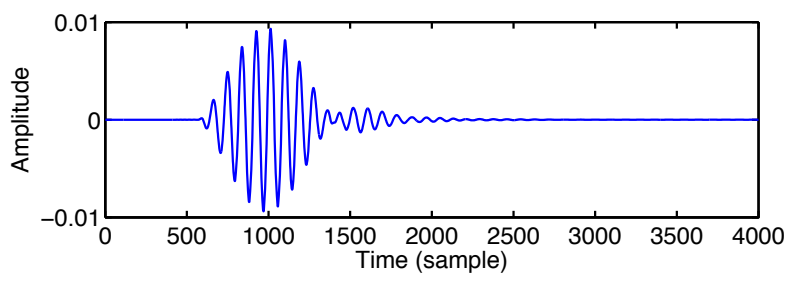

(b) $\mathbf{k}_{t}[n]=\mathbf{k}_{c+t}[n]-\mathbf{k}_{c}[n]$ obtained by direct subtraction

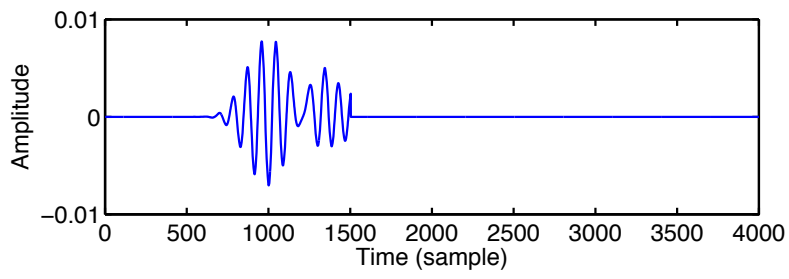

(c) $\mathbf{k}_{t}[n]$ estimated by the DAF/EDF algorithm

Fig. 4: Estimated target response obtained from the DAF/EDF approach for tumour in the middle of the breast tissue.

we align and scale the recorded responses $\mathbf{k}_{c+t}^{(i-1)}$ and $\mathbf{k}_{c+t}^{(i+1)}$ with respect to the peak in $\mathbf{k}_{c+t}^{(i)}$. Note that aligning and scaling only removes the disparity of the channel response at the peak sample for receivers $(i-1, i, i+1)$ but other samples are still not matched. If the receiver $(i-1)$ and $(i+1)$ are close enough to the $i$ 'th receiver, the least mean square (LMS) algorithm can estimate the clutter response for the $i$ 'th receiver in the temporal window $\left[n_{0}, \cdots, n_{0}+m-1\right]$ by finding the best fit for the delay $J$ and magnitude of the clutter. The delay $J$ is an independent variable and is related to the inter-antenna spacing and the sampling frequency, which are both instrumental parameters in the DAF/EDF algorithm. Note that the delay $J$ in the DAF implementation is determined experimentally. Based on delay $J$, the input of the LMS algorithm for finding the FIR filter coefficients, which is called the clutter signature for the $i^{t h}$ receiver $\mathbf{b}_{(i-1)}^{(i+1)}[n]$, is given by

$$
\begin{gathered}
\forall(2 \leq i \leq N-1): \\
\mathbf{b}_{(i-1)}^{(i+1)}[n]=\left[k_{c+t}^{(i-1)}[n-J], \cdots, k_{c+t}^{(i-1)}[n+J],\right. \\
\left.k_{c+t}^{(i+1)}[n-J], \cdots, k_{c+t}^{(i+1)}[n+J]\right]^{T} .
\end{gathered}
$$

3. Filter Coefficients: The coefficients of the filter are given by

$$
\mathbf{q}_{2 i}=\mathbf{R}^{-1} \mathbf{p},
$$




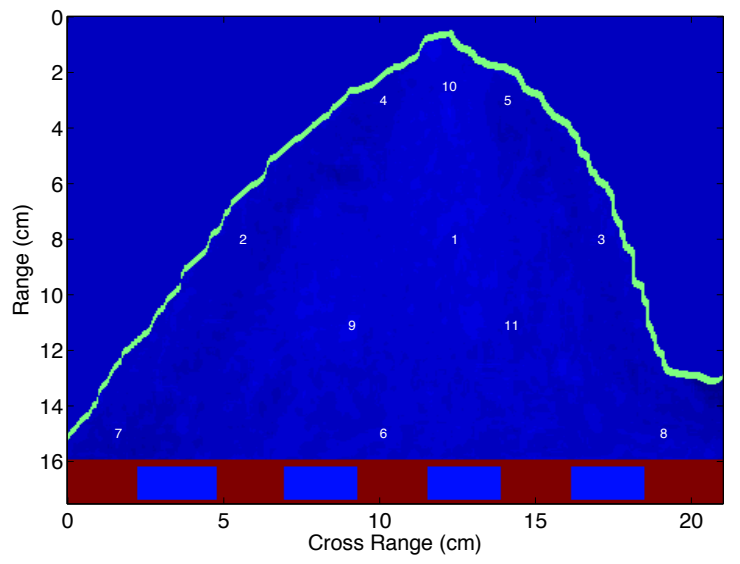

Fig. 5: A spherical $2 \mathrm{~mm}$ cancerous tumour is introduced at eleven different locations marked as 1 to 11 in the figure.

where

$$
\begin{aligned}
\mathbf{R} & =\frac{1}{m} \sum_{n=n_{0}}^{n_{0}+m-1} \mathbf{b}_{(i-1)}^{(i+1)}[n] \mathbf{b}_{(i-1)}^{(i+1)^{T}}[n], \\
\text { and } \quad & \mathbf{p}=\frac{1}{m} \sum_{n=n_{0}}^{n_{0}+m-1} \mathbf{b}_{(i-1)}^{(i+1)}[n] k_{c+t}^{(i)}[n] .
\end{aligned}
$$

Since $R$ contains only the clutter response, the pseudoinverse is calculated based on the nonzero eigenvalues.

4. Tumour Response: Apply the filter coefficients estimated in Step 3 to calculate the tumour response using the relationship

$$
k_{t}^{(i)}[n]=k_{c+t}^{(i)}[n]-\mathbf{q}_{2 i}^{T}[n] \mathbf{b}_{(i-1)}^{(i+1)}[n] .
$$

Because $R$ contains only the clutter response, there is no need to add additive steps to remove artifacts due to the tumour response in $R$ as is required by the data adaptive filter.

As a side note to our discussion, we note that the computational complexity of the proposed DAF/EDF algorithm is the same as the DAF approach [7]. The DAF/EDF algorithm adds two steps (i.e., the Hilbert transform and peak alignment), which have lower complexity as compared to the DAF step in the DAF/EDF algorithm.

Next, the DAF/EDF algorithm is coupled with the TR array imaging approaches to test the performance of the proposed breast cancer detection algorithm.

\section{SIMULATIONS AND RESULTS}

In order to compare the performance of the DAF/EDF algorithm, we use the electromagnetic (EM) finite difference time domain (FDTD) environment to simulate the propagation of the microwave probing signal through breast tissue. The EM model of the breast tissue and the probing signals used are the same as specified in our earlier work [1]. As shown in Fig. 5, the proposed DAF/EDF based TR algorithm is tested by inserting spherical $2 \mathrm{~mm}$ tumours at 11 different locations. Additive White Gaussian observation noise (AWN) is added but the signal-to-noise ratio is maintained at $30 \mathrm{~dB}$. The results for location 1 are being reported in this paper. Fig. 4 compares the

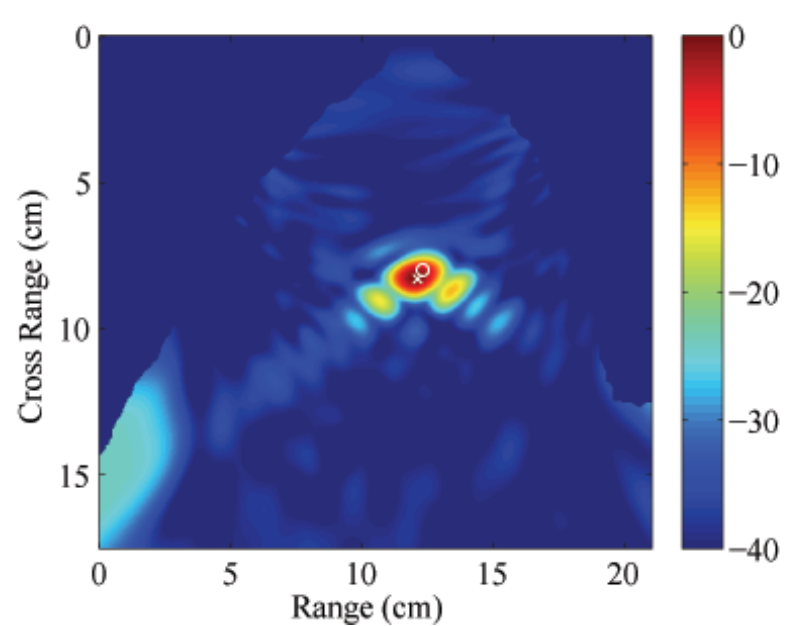

(a) TRAIC/TRBF

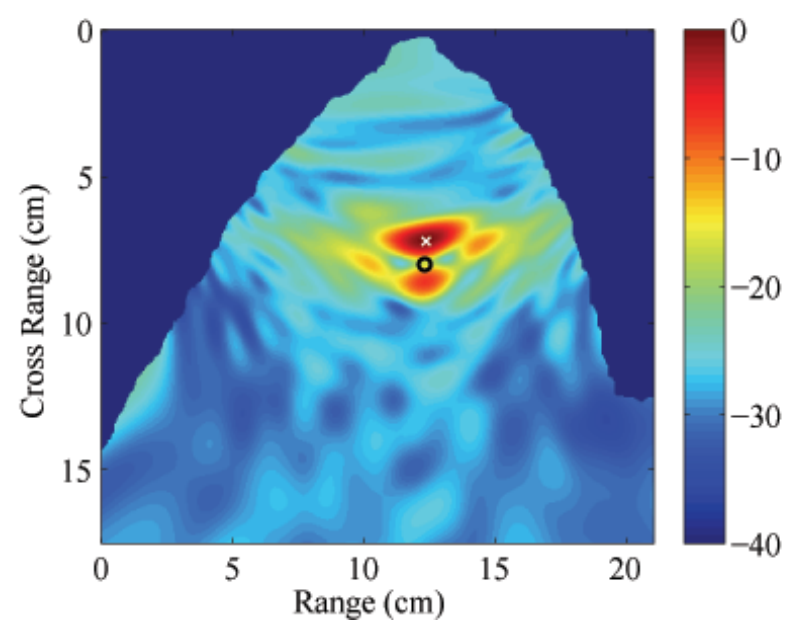

(b) TRAIC/MUSIC

Fig. 6: Array imaging pseudospectrum for location 1 and SNR = $30 \mathrm{~dB}$ obtained from 2 TR array imaging algorithms when tumour response obtained by DAF/EDF algorithm. The tumour location is represented by ' $\circ$ ', while the estimated location is given by ' $x$ '.

output (Fig. 4(b)) of the DAF/EDF algorithm with background subtraction (Fig. 4(c)). Since we have physically inserted the tumour, therefore, we have access to both the tumour-free breast and the one with tumour. Therefore, we can experimentally determine both the overall response $\mathbf{k}_{c+t}[n]$ and the clutter response $\mathbf{k}_{c}[n]$ for each pair of transmitting and receiving antenna. With $A_{1}$ transmitting and $B_{1}$ receiving, the target response $\mathbf{k}_{t}[n]$ shown in Fig. 4(b) is obtained by background subtraction and is the ground truth. Fig. 4(c) is the output of the DAF/EDF algorithm for the same pair $\left\{A_{1}, B_{1}\right\}$ of the antenna arrays. We see that the two estimated target responses are fairly similar in nature. Next, we show that the DAF/EDF estimated target response estimates the tumour location accurately.

Fig. 6 shows that output of the two TR array imaging approaches, namely the TR Adaptive Filter Canceler/Time Reversal Beamforming (TRAIC/TRBF) and the TRAIC/Multiple Signal Classification (TRAIC/MUSIC) as developed in $[1,2,3]$. In both cases, the output 


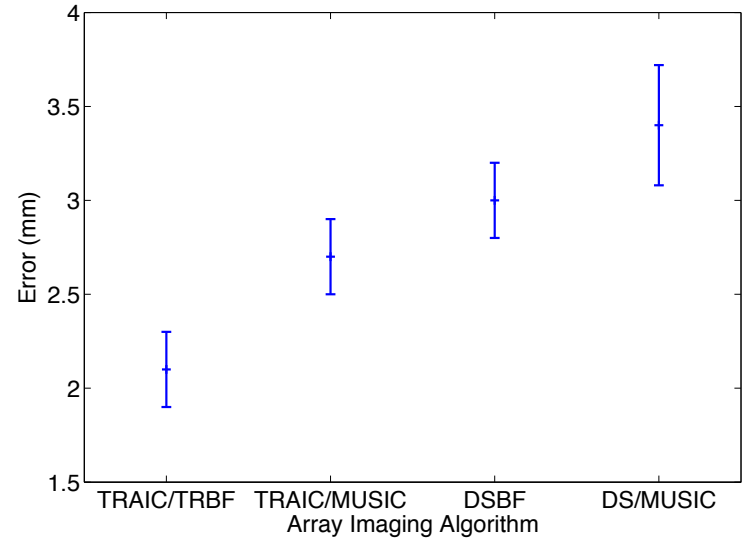

Fig. 7: Range of absolute errors (mean \pm standard deviation) in estimating the location tumour derived by running a Monte-Carlo simulation at location 1 for the four array imaging algorithms for a SNR of $30 \mathrm{~dB}$.

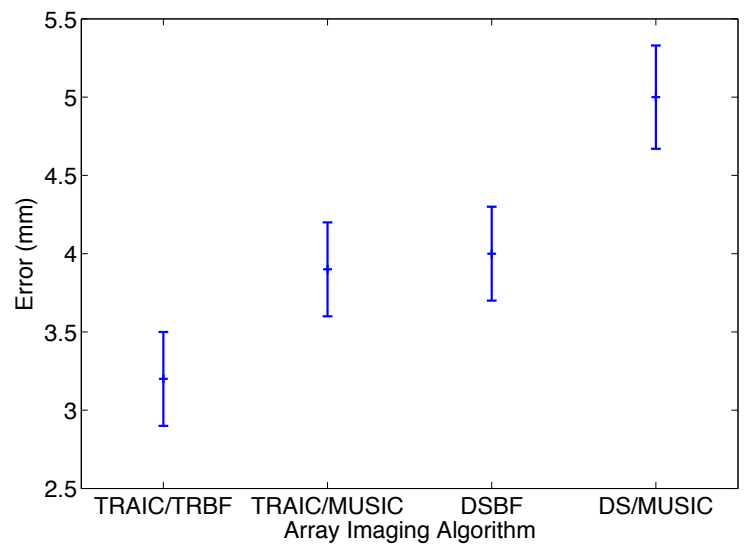

Fig. 8: Same as Fig. 7 except for a SNR of $15 \mathrm{~dB}$.

of the DAF/EDF algorithm is used to determine the target responses. Fig. 6 shows that the tumour is located with a sufficiently high precision. The DAF/EDF algorithm is, therefore, doing a reasonable job in terms of suppressing the clutter and deriving the target response from the overall response.

To test the performance of the performance of the TR approaches with the conventional array imaging approaches such as Direct Subtraction Beam Forming (DSBF) and Direct Subtraction MUSIC (DS/ MUSIC), Figs. 7 and 8 compare the absolute errors for tumour location 1 in the estimated outputs of the four approaches obtained from a Monte Carlo simulation of 1000 runs for tumour location 1 at two different SNRs of $30 \mathrm{~dB}$ and $15 \mathrm{~dB}$. Each vertical line in Figs. 7 and 8 represent the mean error value (shown by the middle value) as well as the value of the errors one standard deviation above and below the mean. In each case, we note that the TR approaches outperform their conventional counterparts. Similar results were noted for other tumour locations.

\section{CONCLUSION}

In this paper, we have shown that the combination of the TR array imaging algorithms and the DAF/EDF algorithm offers a practical approach to detect breast cancer because: $(i)$ it does not need a training stage, and; (ii) it can overcome the multipath distortions commonly observed in microwave breast imaging. Our FDTD simulations show that TR imaging algorithms combined with the DAF/EDF can localize breast tumours with a fairly high accuracy at a variety of SNRs we tested.

\section{REFERENCES}

[1] M. Sajjadieh, F. Foroozan, and A. Asif, "Breast cancer detection using time reversal signal processing," in IEEE 13th International Multitopic Conference (INMIC), pp. 1-5, 2009.

[2] Y. Jin, Y. Jiang, and J.M.F. Moura, "Time reversal beamforming for microwave breast cancer detection," in IEEE International Conference on Image Processing (ICIP), vol. 5, pp. 1316, 2007.

[3] Y. Jin, J.M.F. Moura, Y. Jiang, M. Wahl, H. Zhu, and Q. He, "Breast cancer detection by time reversal imaging," in IEEE International Symposium on Biomedical Imaging: From Nano to Macro (ISBI), vol. 5, pp. 816-819, 2008.

[4] N. Duric, P. Littrup, E. Holsapple, A. Babkin, R. Duncan, A. Kalinin, R. Pevzner, and M. Tokarev, "Ultrasound imaging of breast tissue," in Proceedings of the SPIE: Medical Imaging, pp. 21-26, 2003.

[5] N. Duric, P. Littrup, A. Babkin, D. Chambers, S. Azevedo, A. Kalinin, R. Pevzner, M. Tokarev, E. Holsapple, O. Rama, et al., "Development of ultrasound tomography for breast imaging: Technical assessment," Medical Physics, vol. 32, pp. 1375, 2005.

[6] J.M.F. Moura and Y. Jin, "Time reversal imaging by adaptive interference canceling," IEEE Transactions on Signal Processing, vol. 56, no. 1, pp. 233-247, 2008.

[7] EJ Bond, X. Li, SC Hagness, and BD Van Veen, "Microwave imaging via space-time beamforming for early detection of breast cancer," IEEE Transactions on Antennas and Propagation, vol. 51, no. 8, pp. 1690-1705, 2003.

[8] J.F. Claerbout, Fundamentals of Geophysical Data Processing, Citeseer, 1976.

[9] Kosmas, P. and Rappaport, C.M. A matched-filter FDTD-based time reversal approach for microwave breast cancer detection, IEEE Transactions on Antennas and Propagation, vol. 54, no. 4, pp. 1257-1264, 2006 